\title{
DOES A NUCLEUS ACT LIKE A GLUON FILTER?
}

\section{A. KRZYWICKI}

$Z i F$, University of Biele feld, Bielefeld, Germany

and LPTPE, University of Paris XI, Orsay, France ${ }^{1}$

\section{J. ENGELS and B. PETERSSON}

Department of Physics, University of Bielefeld, Bielefeld, Germany

and

\author{
U. SUKHATME \\ LPTPE, University of Paris XI, Orsay, France
}

Received 4 June 1979

\begin{abstract}
We study large- $p_{\mathrm{T}}$ hadron production off nuclear targets in the framework of the QCD inspired parton model. The anomalous nuclear enhancement is interpreted as being due to multiple scattering of partons. The curious trigger dependence of the enhancement results mainly from a particularly intense multiple scattering of gluons. This is a novel and interesting observable manifestation of the existence of the gluon self-coupling.
\end{abstract}

Since quantum chromodynamics (QCD) is a nonabelian gauge theory of interacting quarks and gluons, its lagrangian involves a self-interaction of the gauge field. The existence of this gluon self-interaction is at the origin of asymptotic freedom, and it is precisely this property which makes QCD a strong candidate for the theory of hadrons. The identification of new observable manifestations of the gluon self-coupling is an exciting challenge for phenomenologists, and this is the main motivation for this work.

If one is searching for manifestations of a specific type of coupling one should concentrate on phenomena where perturbative arguments make sense. This is hopefully the case for "hard" processes, i.e. those involving large invariant momentum transfers. Furthermore, since gluons do not couple to leptons, we shall consider interactions involving hadrons only, and this naturally leads us to study the production of hadrons at large $p_{\mathrm{T}}$ in high-energy hadronic collisions $\left(p_{\mathrm{T}}=\right.$ transverse momentum).

${ }^{1}$ Permanent address. Laboratory associated with CNRS.
In this paper, we shall assume that large $p_{\mathrm{T}}$ production can be described in the framework of perturbative QCD [1-7]. In such a scheme the most important amplitudes contributing to hard scattering involve the single-gluon exchange. A glimpse at the relevant diagrams shows that the ratio of the hard gluon-nucleon and quark-nucleon cross sections is given (roughly) by the colour factor $9 / 4$. Thus, gluons interact more strongly with matter than quarks as a consequence of the existence of the triple-gluon coupling. One can enhance this difference in the interaction strength by putting several scatterers close to each other in space and letting quarks and gluons undergo multiple hard scattering. It follows that one can expect to see the effects of the triple-gluon coupling by carefully analysing the atomic mass number dependence of large- $p_{\mathrm{T}}$ production on nuclei.

It is generally accepted that large- $p_{\mathrm{T}}$ hadronic production off a nucleus comes from the interactions of the fast partons in the projectile. Unlike the "soft" hadron nucleus collisions [8], the "hard" interactions are characterized by short time scales. Since the fast 
partons behave, in a sense, like penetrating radiation, they "see" the whole target and the cross section for a hard interaction is proportional to the volume of the nucleus. Hence, one gets the standard parton model prediction

$\left.\left(E \mathrm{~d} \sigma / \mathrm{d}^{3} p\right)_{\text {hard }}^{\mathrm{h} A}=A\left(E \mathrm{~d} \sigma / \mathrm{d}^{3} p\right)\right)_{\mathrm{hard}}^{\mathrm{hN}}$.

(Here, $A$ denotes both the nucleus and the number of nucleons in it, whereas $\mathrm{N}$ stands for the nucleon.) Multiple scattering effects modify the $A$-dependence of eq. (1), and we shall find that these corrections are often quite large.

Data. Before going further let us briefly review the current experimental situation. Essentially all available data are for proton beams. The inclusive cross section $E \mathrm{~d} \sigma / \mathrm{d}^{3} p$ for the process proton + nucleus $\rightarrow$ hadron + anything increases with increasing $A$ (roughly) like $A^{n}$ and it is found [9-11] that the exponent $n=n\left(p_{\mathrm{T}}\right)$ overshoots unity at large values of the transverse momentum $p_{\mathrm{T}}$ of the trigger hadron ${ }^{\neq 1}$. Furthermore, the exponent $n\left(p_{\mathrm{T}}\right)$ has a curious dependence on the flavour content of the trigger hadron: the production of particles with flavour content very different from that of the projectile is particularly strongly enhanced. Thus $n$ is at most equal to 1.15 for trigger $\pi^{ \pm}$or $\mathrm{K}^{+}$, while one observes values of $n$ around 1.3 for trigger $\mathrm{K}^{-}$ and $\overline{\mathrm{p}}$. (Note that for a tungsten target, a difference of 0.15 in the value of $n$ corresponds to a factor of 2 in the cross section. $)^{\neq 2}$ Large values of $n\left(p_{\mathrm{T}}\right)$ have also been observed in a jet trigger experiment [14]. With incident protons (pions) one observes $n$ as large as about $1.5(1.3)^{\neq 3}$.

We shall now study the large- $p_{\mathrm{T}}$ production of hadrons off nuclear targets in the framework of the QCD-inspired parton model. The "anomalous" ( $n$ $>1$ ) enhancement of the large- $p_{\mathrm{T}}$ production will be a

\#1 A similar behaviour has been observed in the production of hadron pairs $[12,13]$.

\#2 We should mention that one also observes large values of $n$ for a proton trigger. The production of large- $p_{\mathrm{T}}$ protons using a proton beam is a somewhat special case in the phenomenology of large- $p_{\mathrm{T}}$ production and we shall not discuss it here.

\#3 We subtract about 0.2 from $\max (n)$ read in fig. 1 of ref. [14] to take care of systematic errors and ambiguities in the operational definition of a jet (cf. the discussion in ref. [14]). consequence of the multiple scattering of partons ${ }^{\neq 4}$, and we will show that the trigger dependence of the exponent $n\left(p_{\mathrm{T}}\right)$ reflects the existence of the triplegluon coupling.

Multiple scattering formalism. Our formalism is equivalent to that used earlier by Kühn [17] (see also ref. [18]). We proceed naively, treating the incident partons as "true" particles impinging on a target nucleus. The building block of the model is the invariant inclusive cross section $h\left(p \rightarrow p^{\prime}\right)$ for the transition parton + nucleon $\rightarrow$ parton + anything. (For simplicity, the formulae given below are for only one species of partons; the generalization to several species is trivial. The four-momenta of the initial and of the final parton are denoted by $p$ and $p^{\prime}$, respectively.) The parton-nucleus invariant cross section, $H\left(p \rightarrow p^{\prime}\right)$, is developed in a multiple scattering series as follows:

$H\left(p \rightarrow p^{\prime}\right)=A h\left(p \rightarrow p^{\prime}\right)+\sum_{n=2}^{\infty} H_{n}\left(p \rightarrow p^{\prime}\right)$,

where

$$
\begin{aligned}
& H_{n}\left(p_{0} \rightarrow p_{n}\right)=\rho^{n} \int\left(\mathrm{d} p_{1}\right) \ldots\left(\mathrm{d} p_{n-1}\right) \\
& \quad \times h\left(p_{0} \rightarrow p_{1}\right) \ldots h\left(p_{n-1} \rightarrow p_{n}\right) G\left(p_{0}, \ldots, p_{n}\right) .
\end{aligned}
$$

At very high energy and in the laboratory frame

$$
\begin{aligned}
& G\left(p_{0}, \ldots, p_{n}\right)=\int \mathrm{d}^{2} b \int_{z_{0}}^{z_{n+1}} \mathrm{~d} z_{1} \ldots \int_{z_{n-1}}^{z_{n+1}} \mathrm{~d} z_{n} \\
& \quad \times \exp \left[-\rho \sum_{j=0}^{n} \sigma_{j}\left(z_{j+1}-z_{j}\right)\right] .
\end{aligned}
$$

In the above equations $(\mathrm{d} p)=\mathrm{d}^{3} p / E, b$ is the impact vector, $z_{n+1}=-z_{0}=\left(R^{2}-b^{2}\right)^{1 / 2}$ with $R=R_{0} A^{1 / 3}$ and $R_{0}$ set to $1.14 \mathrm{fm}$. We take the nucleus to be a

${ }^{* 4}$ We assume that a nucleus behaves like a collection of weakly bound nucleons in slow relative motion. One of us (A.K.) has suggested [15] that this picture might be wrong when the nucleus is probed during a very short time. The mechanisms of ref. [15] and of this paper are not mutually exclusive. However, the absence of any anomalous nuclear enhancement in $\mu \bar{\mu}$ production by protons [16] indicates that unconventional nuclear effects are of secondary importance for large- $p_{\mathrm{T}}$ production of hadrons. 
sphere of radius $R$ and uniform density $\rho=3 / 4 \pi R_{0}^{3}$. Finally

$\sigma_{j} \equiv \sigma\left(p_{j}\right)=\frac{1}{2} \int\left(\mathrm{d} p^{\prime}\right) h\left(p_{j} \rightarrow p^{\prime}\right)$,

is the total inelastic "hard" cross section. The factor $1 / 2$ appears because the multiplicity of final nonspectator partons equals two in each parton-nucleon collision. Developing the exponential in eq. (4) and collecting terms of the same order in $\sigma$ one finds

$H\left(p \rightarrow p^{\prime}\right) / h\left(p \rightarrow p^{\prime}\right)=A+c_{2} A^{4 / 3}+c_{3} A^{5 / 3}+\mathrm{O}\left(\sigma^{3}\right)$,

where, for example, the coefficient $c_{2}$ is [17]

$$
\begin{aligned}
c_{2} & =\left(9 / 16 \pi R_{0}^{2}\right)\left[\int\left(\mathrm{d} p_{1}\right) \frac{h\left(p \rightarrow p_{1}\right) h\left(p_{1} \rightarrow p^{\prime}\right)}{h\left(p \rightarrow p^{\prime}\right)}\right. \\
& \left.-\sigma(p)-\sigma\left(p^{\prime}\right)\right] .
\end{aligned}
$$

The negative terms in the square brackets correspond to the geometrical shadow effect. In general, this effect is non-negligible and has to be carefully taken into account ${ }^{\neq 5}$. A priori, it is not even clear whether $c_{2}>0$, which is essential for producing an $A$-dependence $A^{n}$ with $n>1$. Thus, the common lore that multiple scattering immediately gives anomalous nuclear enhancement is wrong!

We assume that nuclear matter is exactly iso-scalar, so that $u$ and $d$ quarks scatter alike. Furthermore, we neglect the q $\bar{q}$ sea (but not the gluon sea) in the incident and target nucleons as well as the transition gg $\rightarrow q \bar{q}$ ( $q$ and $g$ stand for "quark" and "gluon", respectively). We checked that our results are only weakly sensitive to this approximation, which simplifies the problem considerably: We have only four distinct transitions, $q \rightarrow q, q \rightarrow g, g \rightarrow q, g \rightarrow g$ and our multiple scattering is a $2 \times 2$ matrix problem. Terms of order $O\left(\sigma^{3}\right)$ in eq. (6) have been estimated, found negligible and neglected. The computations have been done using the Monte-Carlo method.

The cross section $h\left(p \rightarrow p^{\prime}\right)$ is found from the standard recipe of the parton model:

\$5 On this point we disagree with the opinion expressed in ref. [17]. With an input very similar to that of ref. [17] (but with only one intermediate state) we find $c_{2}<0$, which corresponds to an anomalous nuclear reduction.

$$
\begin{gathered}
h\left(a, p_{\mathrm{a}} \rightarrow \mathrm{c}, p_{\mathrm{c}}\right)=\frac{1}{\pi} \sum_{\mathrm{b}} \int \frac{\mathrm{d} x}{x} \mathrm{~d}^{2} p_{\mathrm{b} T} F_{\mathrm{b}}\left(x, p_{\mathrm{b} T}, Q^{2}\right) \\
\times \frac{\mathrm{d} \sigma}{\mathrm{d} t}\left(\mathrm{a}+\mathrm{b} \rightarrow \mathrm{c}+\mathrm{b}^{\prime} ; s, t, u\right) \delta(1+t / s+u / s),
\end{gathered}
$$

where $s=2 p_{\mathrm{a}} \cdot p_{\mathrm{b}}, t=-2 p_{\mathrm{a}} \cdot p_{\mathrm{c}}$ and $u=-2 p_{\mathrm{b}} \cdot p_{\mathrm{c}} ; x$ is the scaled longitudinal momentum of the parton $b$ and $p_{\mathrm{b} T}$ is its transverse momentum. Here, the "longitudinal" direction is defined by the momentum of parton a in the rest frame of $a+N$. Thus, our "smearing" procedure, adapted to the multiple scattering calculation, is slightly different from the usual one. This does not make much difference in practice.

Technicalities. The differential cross sections for QCD processes in the Born approximation can be found in the literature [1-5]. We "regularize" them by adding $1 \mathrm{GeV}^{2}$ to $s,-t$ and $-u$ in the denominators. We define $Q^{2}$ (following ref. [1]) by the equation $Q^{2}$ $=2 s t u /\left(s^{2}+t^{2}+u^{2}\right)$. We also set

$$
F\left(x, p_{\mathrm{T}}, Q^{2}\right)=F\left(x, Q^{2}\right) \exp \left(-p_{\mathrm{T}}^{2} /\left\langle p_{\mathrm{T}}^{2}\right\rangle\right) / \pi\left\langle p_{\mathrm{T}}^{2}\right\rangle,
$$

and we borrow the scale-breaking structure functions $F_{\mathrm{b}}\left(x, Q^{2}\right)$ from ref. [7] (the parameter $\left\langle p_{\mathrm{T}}^{2}\right\rangle$ is set to $\left.0.95(\mathrm{GeV} / c)^{2}\right)$.

Since we take into account the scale breaking in the structure functions, the energy profile of the incident "parton beam" depends on the $Q^{2}$ of the first "hard" collision. Some integrations in eqs. (3) and (8) have to be performed after one has multiplied $H\left(p \rightarrow p^{\prime}\right)$ by the structure function of the incident nucleon. This is easy in a Monte-Carlo calculation.

The exact amount of geometrical scaling depends on the magnitude of the total "hard scattering" cross section, which in turn depends on what is meant exactly by "hard scattering". For our formalism to hold, multiple scattering should involve processes with short time scales only. But since there is no sharp boundary between "hard" and "soft" phenomena, we cannot avoid an ad hoc definition: we call a collision "hard" if $Q^{2}>Q_{\min }^{2}$. The cut-off in $Q^{2}$ should correspond to a cut-off at moderate values of $p_{\mathrm{T}}$, and furthermore $\sigma$ and $\alpha_{s}\left(Q_{\min }^{2}\right)$ should be reasonably small. We set $Q_{\min }^{2}$ $=4 \mathrm{GeV}^{2}$. Without "smearing" this would produce a $p_{\mathrm{T}}$ cut-off near $p_{\mathrm{T}}=1.4 \mathrm{GeV} / c$. The cross section $\sigma_{\mathrm{qN}}\left(\sigma_{\mathrm{gN}}\right)$ rises from $0.17 \mathrm{mb}(0.46)$ at $p_{\mathrm{lab}}=40 \mathrm{GeV} / c$ to $0.86 \mathrm{mb}(2.1 \mathrm{mb})$ at $p_{\text {lab }}=400 \mathrm{GeV} / c$ (the energy interval relevant for the numbers presented in table 1$)$. Finally, we have $\alpha_{s}=12 \pi / 25 \ln \left(Q^{2} / \Lambda^{2}\right)<2 / 3$ with $\Lambda=0.6 \mathrm{GeV}$. 
A nasty point common to all parton model calculations involving $p_{\mathrm{T}}$ "smearing" is that the singularity $x^{-1}$ in the integrand of eq. (8) moves into the integration domain. The standard way out is to replace $x^{-1}$ by $\left[x^{2}+O\left(1 / P^{2}\right)\right]^{-1 / 2}$, where $P$ is the momentum in the NN center-of-mass frame. Different choices of $O\left(1 / P^{2}\right)$ amount to modifications of the structure functions in the wee parton region, where they are badly known. Unfortunately, the multiple scattering does depend on this choice, although rather mildly. Qualitatively, one enhances (reduces) the multiple scattering by taking $O\left(1 / P^{2}\right)$ smaller (larger). We make the reasonable choice $O\left(1 / P^{2}\right)=(100 \mathrm{MeV} / P)^{2}$.

Caveat. It is obvious that the model contains many uncertainties. We have not played with these uncertainties in order to fit the data. We have chosen our input at the outset according to what we felt was a reasonable choice and we stuck to this choice. This attitude has been partly motivated by the complexity of the numerical work and also by the conviction that trifling with parameters would be a futile exercise at the present time. Thus, the numerical results we present should be taken with a grain of salt. They are mainly meant to illustrate and render plausible the qualitative ideas of this paper.
Results and discussion. Let $r(A)$ be the ratio of the cross sections,

$\frac{E \mathrm{~d} \sigma / \mathrm{d}^{3} p(\mathrm{~N}+A \rightarrow \text { parton }+\mathrm{X})}{E \mathrm{~d} \sigma / \mathrm{d}^{3} p(\mathrm{~N}+\mathrm{N} \rightarrow \text { parton }+\mathrm{X})}$,

and let us expand $r(A)$ in powers of $A^{1 / 3}$ as in eq. (6). We work at $90^{\circ}$ in the NN center of mass (as the experimenters). Thus we write

$r(A)=A+r_{2}\left(p_{\mathrm{T}}\right) A^{4 / 3}+r_{3}\left(p_{\mathrm{T}}\right) A^{5 / 3}$.

The results relevant for the anomalous enhancement effect are shown in table 1 . Our values of $E \mathrm{~d} \sigma / \mathrm{d}^{3} p$ $X(\mathrm{NN} \rightarrow$ parton $+\mathrm{X})$ are close to those found by other people (see, e.g. ref. [6] $)^{\ddagger 6}$. The values of $n\left(p_{\mathrm{T}}\right)$ are in quite reasonable agreement with the data, especially those obtained with a single hadron trigger (see also the discussion below). They are significantly lower than $n_{\text {jet }}\left(p_{\mathrm{T}}\right)$ found in ref. [14]. However, the magnitude of $n_{\text {parton }}$ is expected to be smaller than $n_{\text {jet }}$. The hadronization of a quark (gluon) is a soft process and the fast secondary hadrons materialize outside of the

${ }^{\# 6}$ A sample of further results: At $E_{\mathrm{lab}}=400 \mathrm{GeV}$ and $p_{\mathrm{T}}=3$ $\mathrm{GeV} / c,\left(E \mathrm{~d} \sigma / \mathrm{d}^{3} p\right)_{\text {jet }}=3.2 \times 10^{-2} \mathrm{mb} c^{3} \mathrm{GeV}^{-2}$. At 200 $\mathrm{GeV},\left(E \mathrm{~d} \sigma / \mathrm{d}^{3} p\right)_{\mathrm{jet}}=1.4 \times 10^{-2}, 3.0 \times 10^{-5}$ and $3.5 \times 10^{-8}$ $\mathrm{mb} c^{3} \mathrm{GeV}^{-2}$ at $p_{\mathrm{T}}=3,5$ and $7 \mathrm{GeV} / c$, respectively.

Table 1

The figures correspond to an energy of the incident nucleon of $E_{\mathrm{lab}}=400 \mathrm{GeV}$ and to a scattering angle equal to $90^{\circ}$ in the nucleon-nucleon rest frame. The exponent $n$ is here calculated from $n=\log r(A) / \log A$, with $A=184$ (tungsten).

\begin{tabular}{|c|c|c|c|c|}
\hline$p_{\mathrm{T}}(\mathrm{GeV} / c)$ & 5 & 7 & 9 & 11 \\
\hline $\begin{array}{l}E \mathrm{~d} \sigma / \mathrm{d}^{3} \mathrm{p}(\mathrm{NN} \rightarrow \text { quark }) \\
\left(\mathrm{mb} / \mathrm{GeV}^{2}\right)\end{array}$ & $4.13 \times 10^{-5}$ & $5.47 \times 10^{-7}$ & $9.28 \times 10^{-9}$ & $6.96 \times 10^{-11}$ \\
\hline $\begin{array}{l}E \mathrm{~d} \sigma / \mathrm{d}^{3} p(\mathrm{NN} \rightarrow \text { gluon }) \\
\left(\mathrm{mb} / \mathrm{GeV}^{2}\right)\end{array}$ & $1.24 \times 10^{-4}$ & $5.55 \times 10^{-7}$ & $5.90 \times 10^{-9}$ & $3.17 \times 10^{-11}$ \\
\hline $\begin{array}{l}r_{2}\left(p_{\mathrm{T}}\right) \text { for } \\
\text { final quark }\end{array}$ & 0.074 & 0.072 & 0.066 & 0.12 \\
\hline $\begin{array}{l}r_{3}\left(p_{\mathrm{T}}\right) \text { for } \\
\text { final quark }\end{array}$ & -0.0007 & 0.0012 & 0.0006 & 0.0003 \\
\hline $\begin{array}{l}n\left(p_{\mathbf{T}}\right) \text { for } \\
\text { final quark }\end{array}$ & 1.07 & 1.07 & 1.06 & 1.11 \\
\hline $\begin{array}{l}r_{2}(p \mathrm{~T}) \text { for } \\
\text { final gluon }\end{array}$ & 0.23 & 0.35 & 0.27 & 0.47 \\
\hline $\begin{array}{l}r_{3}\left(p_{\mathrm{T}}\right) \text { for } \\
\text { final gluon }\end{array}$ & -0.0035 & 0.0096 & 0.012 & 0.015 \\
\hline$n\left(p_{\mathrm{T}}\right)$ for & 1.15 & 1.23 & 1.21 & 1.27 \\
\hline
\end{tabular}


nucleus. However, slow hadrons are produced within the nucleus and can rescatter. This produces an additional smearing of the jet production cross section and an increase of $n\left(p_{\mathrm{T}}\right)$.

The pattern of multiple scattering reflects the power fall with $p_{\mathrm{T}}$ of the single scattering. The momentum transfers are unequally distributed among the successive collisions. The collisions which "enrich the gluon beam" occur first. The largest momentum transfer usually occurs last $\neq 7$.

A closer look at the calculations shows that both multiple scattering and its geometrical screening are controlled by the single scattering in the $p_{\mathrm{T}}$ region where various non-scaling effects (like the "intrinsic" $p_{\mathrm{T}}$ ) are important. Furthermore, we observe a strong cancellation between the "genuine" triple scattering contribution to $r_{3}\left(p_{\mathrm{T}}\right)$ and the corresponding shadow corrections. Thus, while the magnitude of $r_{2}\left(p_{\mathrm{T}}\right)$ is presumably realistic, only the order of magnitude of $r_{3}\left(p_{T}\right)$ is really meaningful. The coefficient $r_{3}$ could change appreciably with different model parameters. Hence, we feel that the exact value taken by $n\left(p_{\mathrm{T}}\right)$ does not have any great significance. The really interesting point is the dependence of $n\left(p_{\mathrm{T}}\right)$ on whether the final parton is a gluon or a quark.

The considerable difference between $n_{\text {gluon }}$ and $n_{\text {quark }}$ is a consequence of two qualitative features of the theory. First, as anticipated in our introductory remarks, the triple-gluon coupling favours more intense multiple scattering of gluons. Second, the coefficients $r_{j}$ are determined by the ratios of multiple to single scattering cross sections. The inclusive cross section for $\mathrm{N}+\mathrm{N} \rightarrow$ gluon $+\mathrm{X}$ falls steeper with increasing $p_{\mathrm{T}}$, than that for $\mathrm{N}+\mathrm{N}$ quark $+\mathrm{X}$, and this again favours the enhancement of gluon production by multiple scattering. The fall with $p_{\mathrm{T}}$ of the gluon/quark ratio in the process $\mathrm{N}+\mathrm{N} \rightarrow$ parton $+\mathrm{X}$ reflects the different behaviour of gluon and quark structure functions: at large $x$, the structure function of the gluon falls faster with increas-

\#7 Double scattering at $E_{1 \mathrm{ab}}=400 \mathrm{GeV}$ and $p_{\mathrm{T}}=5 \mathrm{GeV} / c$ : first collision at $p_{\mathrm{T}}=2.7 \pm 0.6 \mathrm{GeV} / c$, second collision at $p_{\mathrm{T}}=5.8 \pm 1.3 \mathrm{GeV} / c$ (the number after \pm is the dispersion). Triple scattering: first collision at $p_{\mathrm{T}}=2.5 \pm 0.5$, second at $p_{\mathrm{T}}=2.4 \pm 0.5$ and third at $p_{\mathrm{T}}=5.0 \pm 1.2 \mathrm{GeV} / c$. The corresponding numbers for $p_{\mathrm{T}}=9 \mathrm{GeV} / c$ are: $4.2 \pm 2.0$ and $11 \pm 4.0$ for double scattering and $3.1 \pm 0.7,3.5 \pm 1.0$ and $6.9 \pm 2.0$ for triple scattering. ing $Q^{2}$. This is another consequence of the existence and strength of the gluon self-coupling.

We therefore claim that the particularly intense multiple scattering of gluons is mainly a manifestation of the triple-gluon coupling and is thus a natural prediction of the QCD-inspired parton model. We shall argue now that this effect provides a clue for the understanding of the puzzling trigger dependence of the anomalous nuclear enhancement.

It is generally believed that gluon jets are softer than quark jets. Apart from some theoretical arguments, this belief rests also on indirect phenomenological evidence. Thus, for example, particle ratios on the trigger side would be difficult to understand in the QCD frame work [1] if gluon jets were not more sensitive to the trigger bias [19] than quark jets. Now, as illustrated by our numerical work, gluons undergo a particularly intense multiple scattering and therefore a heavy nucleus acts like a "gluon filter" as far as large $p_{T}$ production is concerned (the fraction of gluon jets is enhanced at large $p_{\mathrm{T}}$ ). Thus, the hierarchy of exponents $n$ can be, at least qualitatively, understood as follows: $n$ is larger when one triggers for a jet instead of a single hadron, because the enhancement is not reduced by the trigger bias. The exponent $n$ for "easily" produced hadrons, like $\pi^{ \pm}$or $\mathrm{K}^{+}$, is close to that for quarks, because the trigger bias drastically reduces the contribution of gluons. A gluon, being flavourless, contributes equally to $\mathrm{K}^{+}$and $\mathrm{K}^{-}$production. However, the gluon contribution to $\mathrm{K}^{+}$production is merely a correction to the larger quark contribution. For $\mathrm{K}^{-}$production, quark (and antiquark) contribution is small. Thus, with the same gluon contribution one gets a strong enhancement for $\mathrm{K}^{-}$and a mild one for $\mathrm{K}^{+}$.

Large- $p_{\mathrm{T}}$ production off nuclei is presumably a privileged place to look for gluon jets. Clearly, we expect jets produced off nuclei to become softer with increasing $A^{\neq 8}$. Also, the correlation between the quantum numbers of the jet and the flavour content of the projectile should weaken. Furthermore, large$p_{\mathrm{T}}$ production off nuclei should be a particularly good place to look for glueballs. At $p_{\mathrm{T}}=7 \mathrm{GeV} / c$ and with a

$\mp 8$ Actually, the authors of the letter quoted in ref. [14] find jets produced off aluminium softer than those produced in hydrogen. We received this issue of Phys. Rev. Lett., when our paper had been almost completed: what would have been a prediction turned into a "postdiction". 
nucleon target, one out of two produced partons is a gluon. This proportion becomes almost three out of four with a uranium target, if one uses our values of $n$. The experimentally observed values of $n$ might suggest a better efficiency of the "filter": if $n_{\text {gluon }} \approx n_{\text {jet }}$ $\approx 1.5$ and $n_{\text {quark }} \approx n_{\text {pion }} \approx 1.1$, one expects nine out of ten jets produced on uranium to be gluon jets (in the appropriate $p_{\mathrm{T}}$ range). This would be a true gluon factory!

One of us (A.K.) is indebted to the Zentrum für interdisziplinäre Forschung for kind hospitality.

\section{References}

[1] R.D. Field, Phys. Rev. Lett. 40 (1978) 997; R.P. Feynman, R.D. Field and G.C. Fox, Phys. Rev. D18 (1978) 3320.

[2] B.L. Combridge, J. Kripfganz and J. Ranft, Phys. Lett. $70 B(1977) 234$.

[3] R. Cutler and D. Sivers, Phys. Rev. D17 (1978) 196.

[4] A.P. Contogouris, R. Gaskell and S. Papadopoulos, Phys. Rev. D17 (1978) 2314.

[5] J.F. Owens, E. Reya and M. Glück, Phys. Rev. D18 (1978) 1501.
[6] J.F. Owens, Florida State Univ. preprint, FSU-HEP790122 (1979).

[7] R. Baier, J. Engels and B. Petersson, Univ. of Bielefeld preprint BI-TP 79/10, to be published in Z. Phys. C.

[8] W. Busza, in: Proc. XIIth Recontre de Moriond (Editions Frontieres, Paris, 1977);

J.D. Bjorken, in: Current induced reactions (Springer, New York, 1976);

A. Capella and A. Krzywicki, Phys. Rev. D18 (1978) 3357.

[9] J.W. Cronin et al., Phys. Rev. D11 (1975) 3105;

L. Kluberg et al., Phys. Rev. Lett. 38 (1977) 670;

D. Antreasyan et a1., Phys. Rev. D19 (1979) 764.

[10] U. Becker et al., Phys. Rev. Lett. 37 (1976) 1731.

[11] D.A. Garbutt et al., Phys. Lett. 67B (1977) 355.

[12] R.L. McCarthy et al., Phys. Rev. Lett. 40 (1978) 213.

[13] D.A. Finley et al., Phys. Rev. Lett. 42 (1979) 1031.

[14] C. Bromberg et al., Proc. VIIIth Intern. Symp. on Multiparticle dynamics (Kaysersberg, 1977); Phys. Rev. Lett. 42 (1979) 1202.

[15] A. Krzywicki, Phys. Rev. D14 (1976) 152; Proc. VIIth Intern. Symp. on Multiparticle dy namics (Tutzing, 1976).

[16] K.J. Anderson et al., Chicago-Princeton Collab., preprint EFT 79-6 (1979).

[17] J.H. Kühn, Phys. Rev. D13 (1976) 2948.

[18] C. Michael and D.M. Webber, Univ. of Liverpool preprint (Feb. 1979).

[19] E. Ellis, M. Jacob and P.V. Landshoff, Nucl. Phys. B108 (1976) 93. 